\title{
Micropatterned Reactive Nanofibers: Facile Fabrication of a Versatile Biofunctionalizable Interface
}

\author{
Ozlem Ipek Kalaoglu-Altan, ${ }^{1}$ Rana Sanyal, ${ }^{1,2}$ and Amitav Sanyal ${ }^{1,2 *}$ \\ ${ }^{1}$ Department of Chemistry, Bogazici University, Bebek 34342, Istanbul, Turkey \\ ${ }^{2}$ Center for Life Sciences and Technologies, Bogazici University, 34342, Istanbul, Turkey \\ E-Mail:amitav.sanyal@boun.edu.tr
}

\section{CONTENTS:}

Measurements and Characterization.

Table S1. Characterizations of copolymers

Figure S1. ${ }^{1} \mathrm{HNMR}$ spectrum of poly(MMA-FuMaMA).

Figure S2. ${ }^{1} \mathrm{HNMR}$ spectrum of poly(MMA-MaMA).

Figure S3. SEC traces of A) poly(PEGMEMA-MMA-FuMaMA) B) poly(PEGMEMAMMA-MaMA) C) poly(MMA-FuMaMA) D) poly(MMA-MaMA). 
Figure S4. SEM images and histograms of poly(PEGMEMA-MMA-FuMaMA) nanofibers A,C) before crosslinking, and B,D) after crosslinking.

Measurements and Characterization. ${ }^{1} \mathrm{HNMR}$ spectra of the polymers were recorded on a Varian $400 \mathrm{MHz}$ nuclear magnetic resonance spectrometer using trimethylsilane as an internal reference and deuterated chloroform as a solvent. The molecular weights of the copolymers were estimated by gel permeation chromatography (GPC) using a PSS-SDV (length/ID $8 \times 300$ $\mu \mathrm{m}, 10 \mu \mathrm{m}$ particle size) mixed-M column calibrated with polystyrene standards $(1-150 \mathrm{kDa})$. A refractive-index detector was used for analysis. Tetrahydrofuran (THF) was used as the eluent at a flow rate of $1 \mathrm{ml} / \mathrm{min}$ at $30{ }^{\circ} \mathrm{C}$. Nanofiber morphology were observed with Jeol Neoscope JCM-5000 scanning electron microscope (SEM). Fluorescence images of samples were recorded at room temperature on a Zeiss Observer Z1 fluorescence microscope. UV light (365 $\mathrm{nm}, 100 \mathrm{~W}$ ) was used for photopatterning. Static water contact angle were measured in air via the sessile-drop method using a goniometer (CAM $101 \mathrm{KSV}$ instruments). Approximately $5 \mu \mathrm{L}$ of deionized water was deposited on the surface and images were taken by an integrated digital camera. The software CAM2008 was used for image processing to determine the contact angle. Contact angle value for each sample was independently measured at three different locations and average values were measured. UV/vis spectroscopy experiments for the quantification of the oligonucleotides were done on a Nanodrop 2000 (Thermo Scientific, USA) instrument. Confocal microscopy images were taken using a Leica TCS SP5 Confocal Microscope and processed using LAS X 3.02 software. 
Table S1. Characterizations of copolymers

\begin{tabular}{cccc} 
Polymer & $\begin{array}{c}\text { Mol \% } \\
\text { PEGMEMA:MMA: } \\
\text { FuMaMA (MaMA) }\end{array}$ & $\mathbf{M}_{\mathbf{n}}(\mathbf{k D a})^{\mathbf{c}}$ & $\mathbf{M}_{\mathbf{w}} / \mathbf{M}_{\mathbf{n}}$ \\
\hline poly(PEGMEMA-MMA-FuMaMA) $^{\mathrm{a}}$ & $20: 60: 20$ & 19.7 & 1.84 \\
poly(MMA-FuMaMA) $^{\mathrm{b}}$ & $0: 80: 20$ & 17.4 & 2.12 \\
poly(PEGMEMA-MMA-MaMA) $^{\mathrm{a}}$ & $20: 60: 20$ & 18.1 & 1.88 \\
poly(MMA-MaMA) $^{\mathrm{b}}$ & $0: 80: 20$ & 16.2 & 2.14 \\
\hline
\end{tabular}

${ }^{\text {a }}$ Polymerizations were performed in THF at $65^{\circ} \mathrm{C},[\mathrm{M}] /[\mathrm{I}]=200$ using V-501 as the initiator. ${ }^{\mathrm{b}}$ Deprotection reactions were performed in toluene at $110^{\circ} \mathrm{C} .{ }^{\mathrm{c}}$ Determined by SEC analysis in THF.

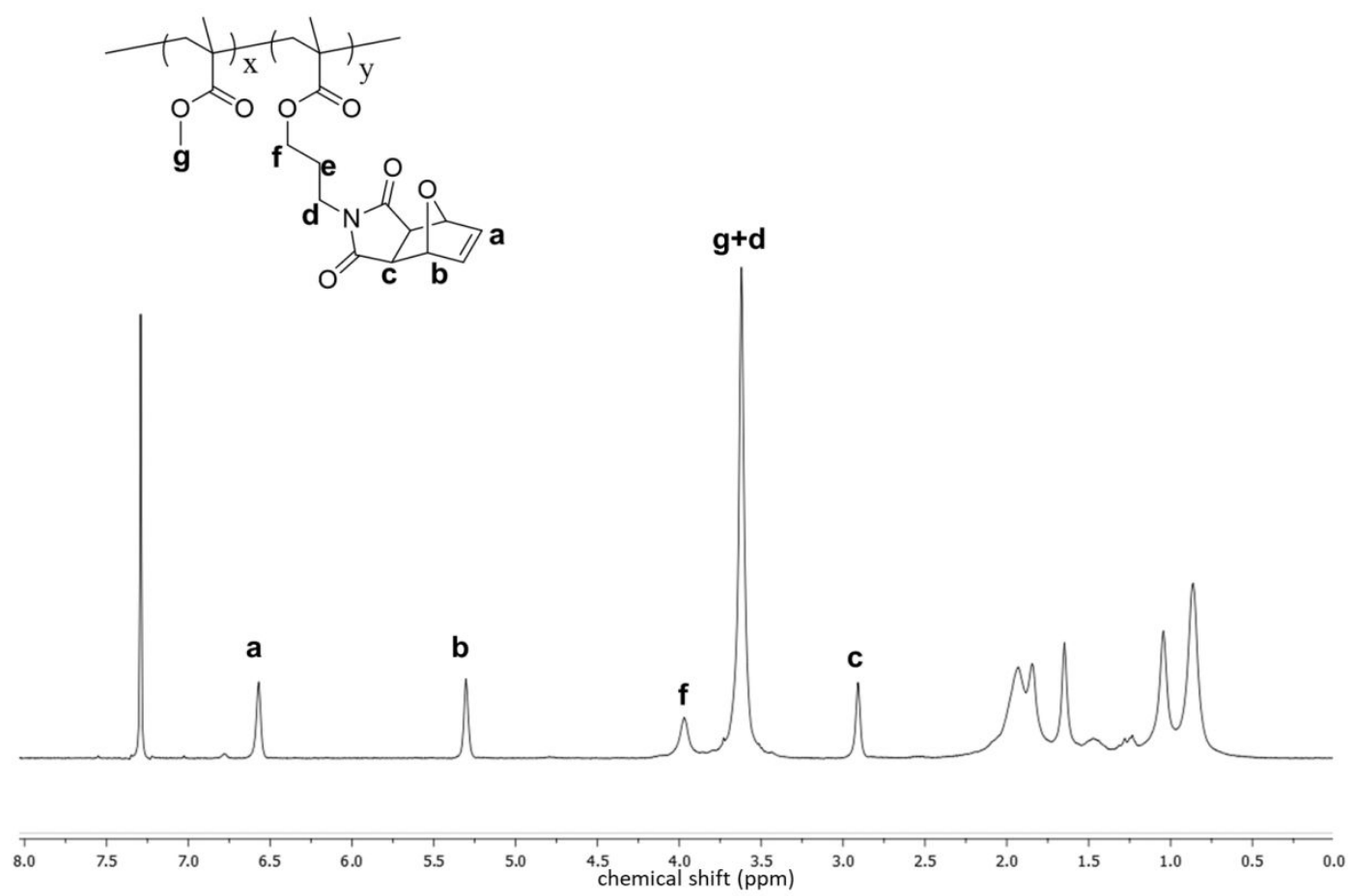

Figure S1. ${ }^{1}$ HNMR spectrum of poly(MMA-FuMaMA) 


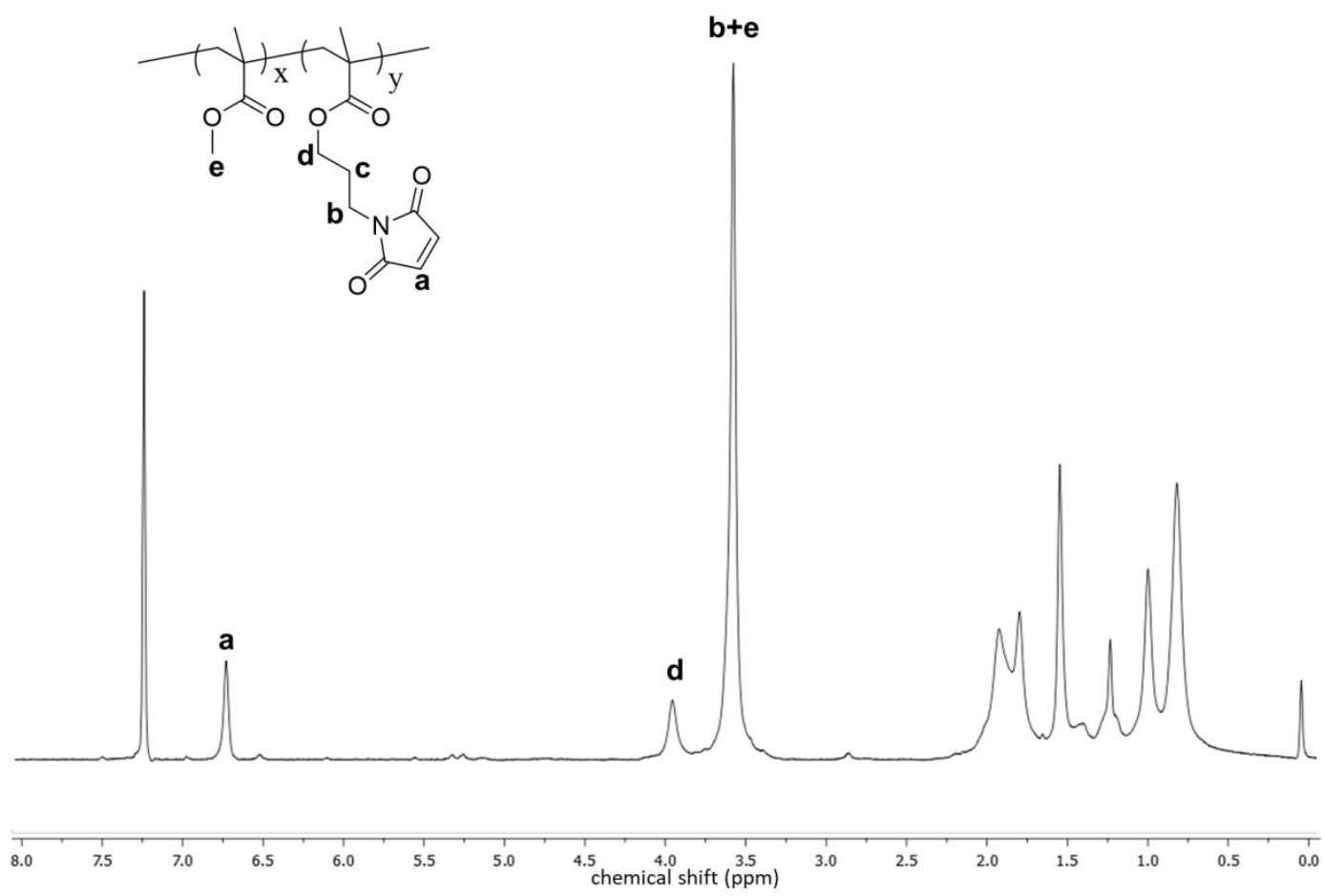

Figure S2. ${ }^{1} \mathrm{HNMR}$ spectrum of poly(MMA-MaMA)
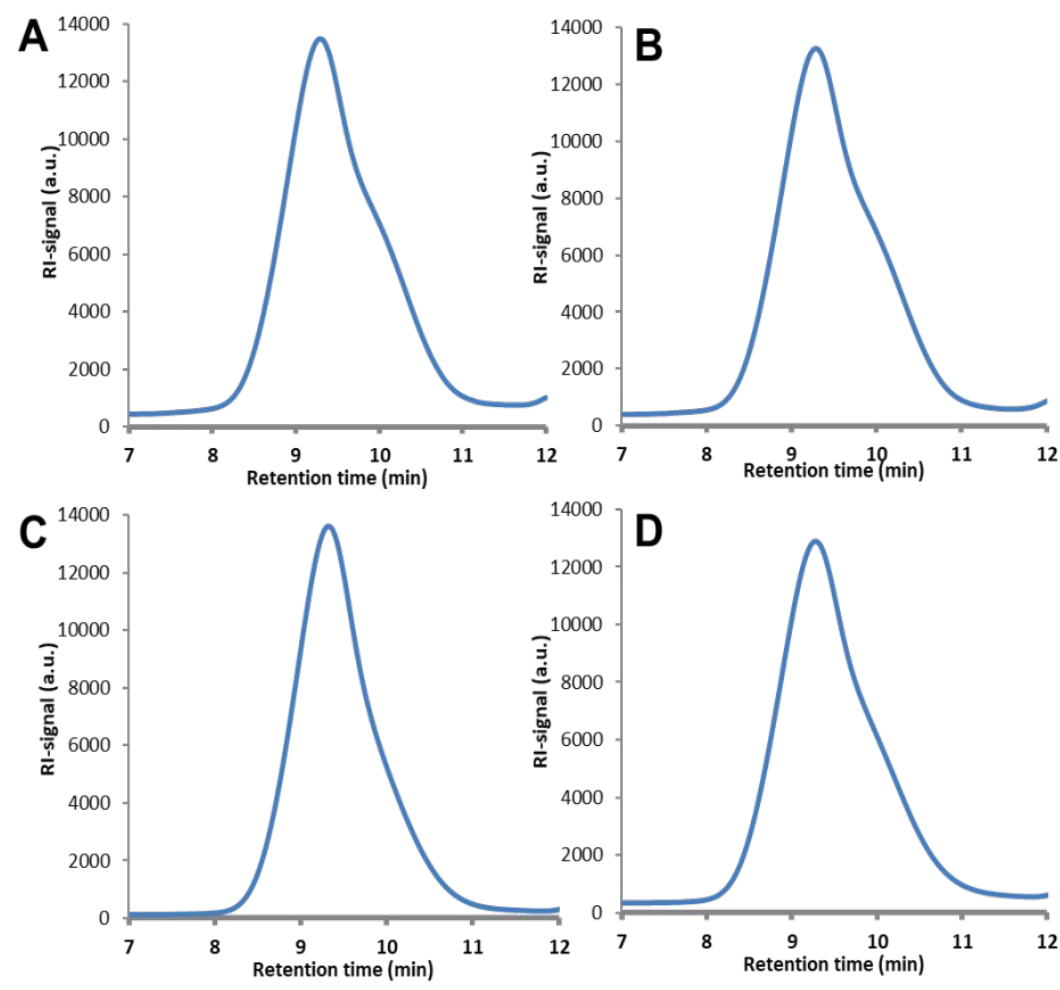

Figure S3. SEC traces of A) poly(PEGMEMA-MMA-FuMaMA) B) poly(PEGMEMAMMA-MaMA) C) poly(MMA-FuMaMA) D) poly(MMA-MaMA). 

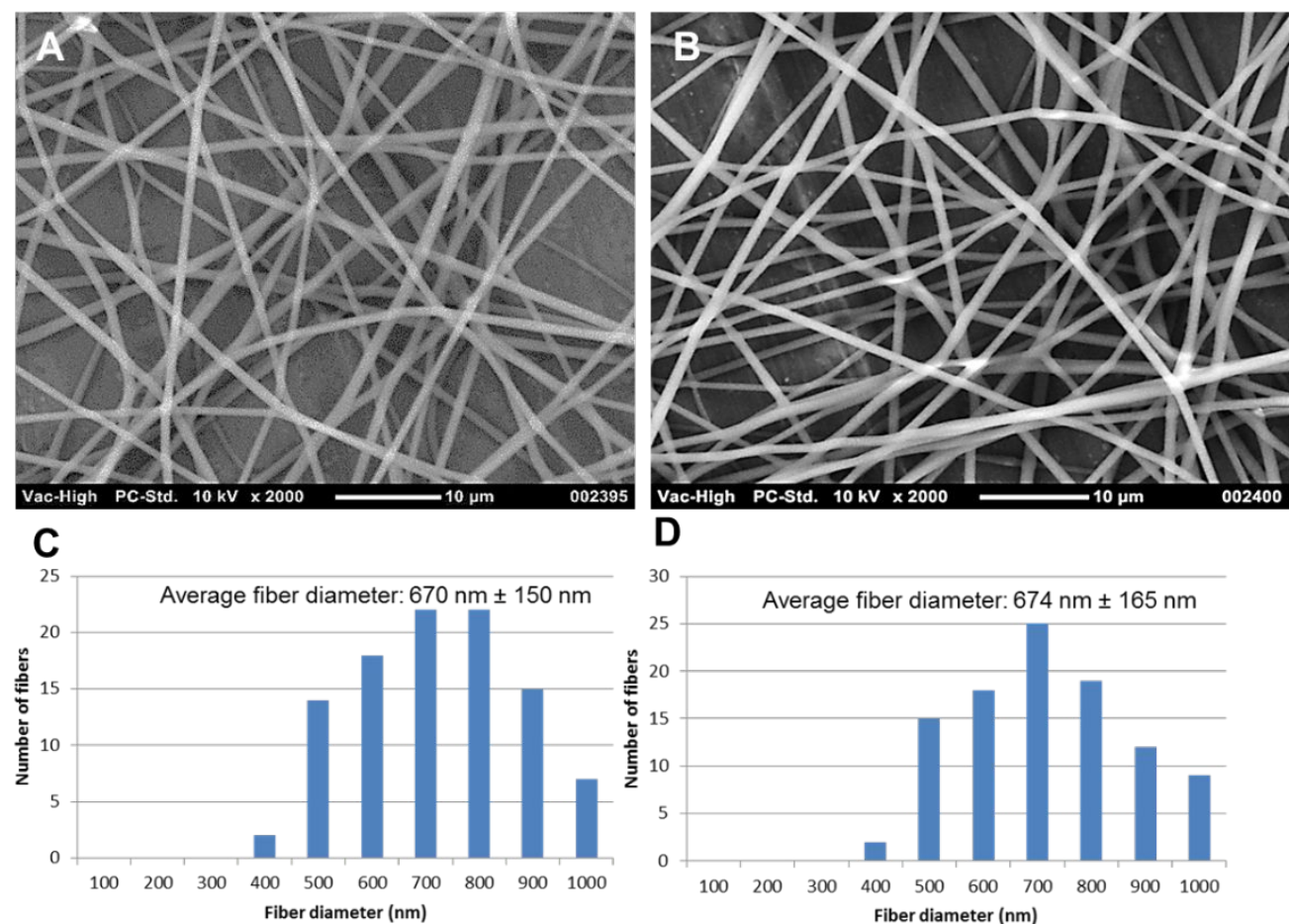

\section{D}

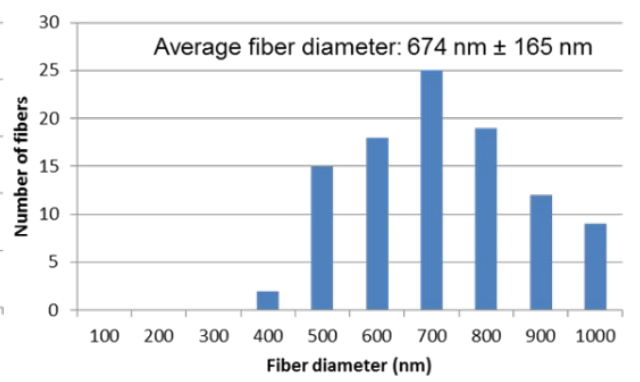

Figure S4. SEM images and histograms of poly(PEGMEMA-MMA-FuMaMA) nanofibers A,C) before crosslinking, and B,D) after crosslinking. 